\title{
On-unit CT measures as tools for artificial intelligence to identify random vs. systematic anatomical changes in radiotherapy patients
}

Nabhya Harjai ${ }^{1}$, Sarah Weppler ${ }^{2}$, Craig A. Beers ${ }^{2}$, Lukas Van Dyke ${ }^{2}$, Colleen Schinkel ${ }^{2,3}$, Wendy Smith ${ }^{2,3}$, Harvey Quon ${ }^{3,4}$

${ }^{1}$ Cumming School of Medicine, University of Calgary.

2 Department of Medical Physics, Tom Baker Cancer Centre

${ }^{3}$ Department of Oncology, University of Calgary

${ }^{4}$ Department of Radiation Oncology, Tom Baker Cancer Centre

\begin{abstract}
Although head and neck (H\&N) cancer survival is steadily increasing, the close proximity of tumor volumes to organs at risk (OARs) makes radiotherapy planning and delivery challenging for these patients. Changes in patient anatomy (i.e. weight-loss, tumor shrinkage) over 7 weeks of daily radiotherapy may result in increased dosages of radiation to OARs relative to the original treatment plan, consequently hindering post-treatment quality of life. Artificial intelligence-based approaches can improve prediction and monitoring of these effects through identification of systematic changes. To collect and perform an analysis of on-unit CT measurements as surrogate measures of dose changes. Correlations among CT measures may indicate random vs. systematic changes in dose deposition (i.e. dosimetry) and further improve artificial intelligence-based approaches that determine which patients benefit most from treatment re-planning. Two hundred and fifty $\mathrm{H} \& \mathrm{~N}$ cancer patients treated with curative chemoradiotherapy were retrospectively analyzed. Five CT measures including face and neck diameter, chin and shoulder position, and head shift were calculated motivated by current literature. Dosimetric changes were calculated for OARs (pharyngeal constrictor, brainstem, parotid and submandibular glands) and tumour volumes. Conventional correlation analysis and hierarchical clustering were performed to assess group-wise correlations. K-medoid clustering and principal components analysis were conducted to infer groupings of the patients as random or systematic. There is a positive correlation between increased dosages to central-axis anatomical structures (spinal cord, pharyngeal constrictor, submandibular glands) and systematic weight-loss effects (change in BMI and weight loss through the face and neck). In line with current literature, clustering indicated that $30.4 \%$ of the cohort exhibited systematic anatomical changes, potentially correctable by re-planning. MANOVA confirmed that the systematic anatomical changes corresponded to the spinal cord and brain stem $(p<0.005)$, and Mann-Whitney $U$ tests confirmed that the measures could identify systematic dose increases to the pharyngeal constrictor $(p<0.05)$. On-unit CT measures appear to be able to distinguish random and systematic dosimetric effects, correlated with changes in dose as expected. These measures can be utilized to improve artificial intelligence-based patient monitoring and intervention techniques.
\end{abstract}

Cite as: Harjai N, Weppler S, Beers CA, Van Dyke L, Schinkel C, Smith S, Quon H. 2019. On-unit CT measures as tools for artificial intelligence to identify random vs. systematic anatomical changes in radiotherapy patients. Alberta Academic Review, Vol 2 (3) 9, CASCH Special Issue (not peer-reviewed), DOI: 10.29173/aar98. 